\title{
Sensitivity of Simulated Transcranial Ultrasound Fields to Acoustic Medium Property Maps
}

\author{
James Robertson, Eleanor Martin, Ben Cox, Bradley E Treeby \\ Dept. Medical Physics and Biomedical Engineering, University College London, Gower \\ Street,London,WC1E 6BT \\ E-mail: james.robertson.10@ucl.ac.uk
}

\begin{abstract}
.
High intensity transcranial focused ultrasound is an FDA approved treatment for essential tremor, while low-intensity applications such as neurostimulation and opening the blood brain barrier are under active research. Simulations of transcranial ultrasound propagation are used both for focusing through the skull, and predicting intracranial fields. Maps of the skull acoustic properties are necessary for accurate simulations, and can be derived from medical images using a variety of methods. The skull maps range from segmented, homogeneous models, to fully heterogeneous models derived from medical image intensity. In the present work, the impact of uncertainties in the skull properties is examined using a model of transcranial propagation from a single element focused transducer. The impact of changes in bone layer geometry and the sound speed, density, and acoustic absorption values is quantified through a numerical sensitivity analysis. Sound speed is shown to be the most influential acoustic property, and must be defined with less than $4 \%$ error to obtain acceptable accuracy in simulated focus pressure, position, and volume. Changes in the skull thickness of as little as $0.1 \mathrm{~mm}$ can cause an error in peak intracranial pressure of greater than $5 \%$, while smoothing with a $1 \mathrm{~mm}^{3}$ kernel to imitate the effect of obtaining skull maps from low resolution images causes an increase of over $50 \%$ in peak pressure. The numerical results are confirmed experimentally through comparison with sonications made through $3 \mathrm{D}$ printed and resin cast skull bone phantoms.
\end{abstract}

\section{Introduction}

The use of ultrasound for neurological applications is an area of active research due to the development of MRI compatible, multi-element arrays that allow effective control and monitoring of intracranial acoustic fields. High intensity focused ultrasound (HIFU) was recently approved by the FDA for ablative treatment of essential tremor in the USA [1, 2]. Developing low intensity applications include opening of the blood-brain barrier (BBB) and direct ultrasonic neuromodulation and stimulation (UNMS). Both techniques have recently been extended to human subjects and are under active research [3,4]. The biggest challenge to ultrasonic brain therapy is the presence of the skull, which aberrates and attenuates incident wavefronts, and reduces the effectiveness of geometrical focusing [5]. Using frequencies $\leq 500 \mathrm{kHz}$ can reduce this distortion [6], however this also increases the focal spot size and the influence of standing waves. The ability of bone to support shear waves can also affect transcranial transmission, although the changes to the intracranial field are typically negligible for ultrasound applied at normal or near-normal incidence $[7,8]$.

To counteract the effect of the skull, model-based aberration correction is often used. This works by simulating the propagation of ultrasound waves through a map of the individual patient's 


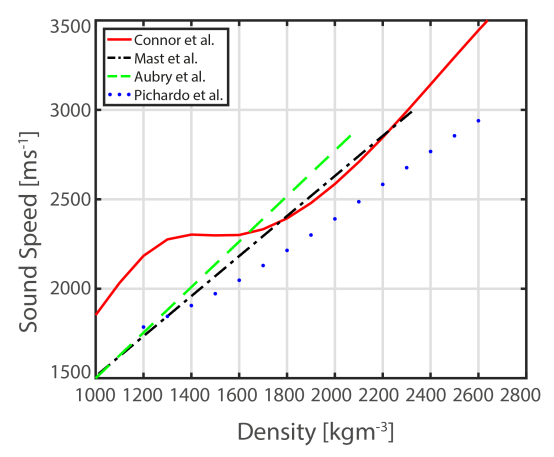

Figure 1: Relationship between the density of the skull bone, derived from CT, and the compressional sound speed. Values taken from Connor et al. [19], Mast et al. [20], Aubry et al. [5], and Pichardo et al. [21].

anatomy, and using the results to correct the drive signals used during therapy. As an example, timereversal (TR) for transcranial focusing is able to correct for aberration by taking advantage of the time-symmetry of the lossless acoustic wave equation through reversing the simulated propagation of ultrasound from a deep brain target to a virtual transducer [5]. In addition to aberration correction, numerical simulations of transcranial propagation are used to predict the intracranial field during the therapy and improve understanding of therapeutic mechanisms $[3,9,10]$.

The accuracy of these predictions, and the effectiveness of model-based aberration correction, is directly dependent on the accuracy of the numerical simulations. A potentially significant source of error is the geometry and acoustic properties of the skull used in the simulated domain. Multiple methods can be used to derive the skull properties from medical image data, with the simplest being segmented, homogeneous models. It is also possible to derive heterogeneous models based on medical image intensity. For example, Aubry et al. [5] developed a widely used technique for the direct conversion of x-ray CT images into maps of skull density, sound speed, and absorption. Absorption values are inflated to imitate the effect of scattering \& reflective losses in the skull layer, which are the dominant attenuating effects [11]. This technique has been used in multiple studies, including a modification using a homogeneous absorption map [12, 13]. Several other models based on empirical relationships between CT derived density and acoustic sound speed and absorption have been also proposed [14]. However, there is some inconsistency in the relationships between sound speed and density, as shown in Fig. 1. MRI based methods, including segmentation [15], and morphological adaptation of virtual CT images have also been examined [16].

The level of fidelity in the acoustic property maps required for accurate simulations is currently unclear [17]. Mueller et al. [18] recently examined the impact of modelling brain and meningeal geometry on simulated intracranial fields, concluding that it is not necessary to model detailed brain tissue geometry. However, given the strong influence of the skull on the propagation of ultrasound, a thorough examination of the sensitivity of intracranial fields to the parameters of the bone tissue map is necessary. This is of special importance for developing, low intensity applications, where tight focusing and fine control over the pressure signal at the target are necessary to achieve the desired effects in the targeted volume, avoid tissue damage, and allow research into the underlying physical mechanisms.

In the present paper, the influence of variation in the acoustic medium properties of the skull on the accuracy of simulated transcranial fields is examined. A numerical forward model of ultrasound 


\begin{tabular}{cccccc}
\hline & Grid Size & Spatial Step & Time Step & Sim. Length & Runtime \\
\hline Fluid 3D & $512 \times 512 \times 1024$ & $145.4 \mu \mathrm{m}$ & $5.1 \mathrm{~ns}$ & $171 \mu \mathrm{s}$ & $19 \mathrm{hr} 53 \mathrm{~min}$ \\
Elastic 2D & $1728 \times 2592$ & $57.2 \mu \mathrm{m}$ & $1.0 \mathrm{~ns}$ & $170 \mu \mathrm{s}$ & $3 \mathrm{hr} 47 \mathrm{~min}$ \\
\hline
\end{tabular}

Table 1: Simulation parameters used for the sensitivity analysis. Fluid simulations were conducted with a minimum spatial sampling of 10 points per wavelength (PPW), and elastic simulations with 25 PPW.

transmission from a single element transducer through a skull bone layer to the brain is constructed. The sensitivity of the intracranial field to changes in the medium properties and geometric parameters of the layer is examined. This numerical sensitivity analysis is then substantiated through the comparison of practical sonication experiments of the transmission of ultrasound through 3D printed and resin cast skull bone phantoms with accurately registered simulations of the sonication setup.

\section{Methods}

\subsection{Simulation Setup used for the Sensitivity Analysis}

To carry out the numerical sensitivity analysis, a virtual model of transcranial ultrasound propagation was created using the open-source k-Wave toolbox [22]. A single element transducer with a radius of curvature (ROC) of $75 \mathrm{~mm}$ and a diameter of $60 \mathrm{~mm}$ was placed $27.3 \mathrm{~mm}$ from a curved layer of homogeneous bone tissue within the simulated environment. This was defined as a $6.5 \mathrm{~mm}$ thick layer taken from the surface of a sphere of $80 \mathrm{~mm}$ radius, in order to approximate the geometry of human skull bone [23]. The transducer geometry was chosen to match the HIFU transducer used in the experimental sonications in Section 2.6, and is similar to the low frequency single element transducers previously used in studies of neuromodulation in humans [9, 24].

The reference medium properties assigned to the bone layer were based on values obtained by White et al. [7] for whole skull compressional and shear wave transmission. Bone compressional speed was $2850 \mathrm{~m} \mathrm{~s}^{-1}$ and density was $1732 \mathrm{~kg} \mathrm{~m}^{-3}$. Absorption values were assigned using power law factors derived from this data, where $a_{0}=8.83 \mathrm{~dB} \mathrm{MHz}^{-\mathrm{y}} \mathrm{cm}^{-1}$ and $y=1.43$ [25]. When simulated, the sound speed of shear waves in the bone layer was set to $1500 \mathrm{~m} \mathrm{~s}^{-1}$, and the absorption power law prefactor was $a_{0}=19.5 \mathrm{~dB} \mathrm{MHz}^{-\mathrm{y}} \mathrm{cm}^{-1}[7]$. The remainder of the simulation domain was assigned the acoustic properties of water, with $c_{\mathrm{w}}=1481 \mathrm{~m} \mathrm{~s}^{-1}$ and $\rho_{\mathrm{w}}=1000 \mathrm{~kg} \mathrm{~m}^{-3}$.

During simulations, the virtual transducer was excited with continuous wave $(\mathrm{CW})$ signals at ultrasonic frequencies of $500 \mathrm{kHz}, 750 \mathrm{kHz}$ and $1 \mathrm{MHz}$. These correspond to a range of frequencies demonstrated effective for transcranial HIFU, UNMS, and ultrasound mediated BBB opening [12]. Once the simulation had reached a steady state, the maximum pressure was recorded across the 3D field following transmission through the bone layer. This was used to evaluate the impact of changes to the bone layer on the intracranial field.

The simulations were performed using both the fluid and elastic codes within the k-Wave toolbox $[26,27]$. The fluid code was used for most simulations, with the elastic code used to confirm that shear waves could be neglected. The medium density, sound speed, and absorption were set to be heterogeneous, and only linear wave propagation was considered (even for high intensity applications, the wave field is generally linear at the skull surface [28]). Elastic wave simulations were conducted in 2D, while fluid simulations were conducted in 3D. Simulation parameters are shown in Table 1. The spatial and temporal discretisations used conform to previously established criteria to ensure numerical accuracy for transcranial simulation [29, 30], with a minimum spatial sampling step of ten spatial points per wavelength $(\mathrm{PPW})$ at $1 \mathrm{MHz}$ for fluid simulations, and $25 \mathrm{PPW}$ for elastic simulations. Fluid simulations were run using the MPI version of k-Wave on the IT4I Salomon 
supercomputing cluster [26]. Each 3D simulation was carried out on a compute node with an 8-core Intel Xeon E5-4627v2 $3.3 \mathrm{GHz} \mathrm{CPU}$, and $256 \mathrm{~GB}$ of RAM. A reference simulation and 567 test simulations were carried out for each frequency.

\subsection{Error Metrics}

Errors in the intracranial fields due to changes in the skull map were quantified relative to a reference simulation. Three error metrics were used: the change in the peak focal pressure, the change in the $-6 \mathrm{~dB}$ volume of the pressure focus (calculated using the number of voxels with pressure amplitude above $50 \%$ of the peak pressure), and the spatial deviation of the centre of mass of the focal volume. Errors in peak pressure of less than $5 \%$ and error in focus position of less than $1.5 \mathrm{~mm}$ were used as criteria for accurate simulation, based on a previous examination of the parameters desirable to achieve effective acoustic neurostimulation [29, 30]. The importance of focal volume will vary according to target, frequency, and transducer characteristics, but an error of less than $20 \%$ was considered appropriate.

\subsection{Effect of Shear Waves in the Skull}

The simulation setup was first used to determine whether modelling shear wave propagation in the skull layer was required. Due to the increased computational burden of modelling elastic wave propagation, and the finer spatial discretisation required [30], the 3D simulation setup was translated into 2D. Simulations were carried out using the k-Wave 2D elastic PSTD code for $500 \mathrm{kHz}, 750 \mathrm{kHz}$ and $1 \mathrm{MHz}$ ultrasound [27]. Two simulations were carried out at each frequency: a fluid simulation with shear speed set to zero throughout the domain, and an elastic simulation with appropriate shear speed and absorption values assigned to the bone layer.

\subsection{Sensitivity to the Acoustic Properties of the Skull}

The sensitivity of the intracranial field to changes in the acoustic properties of the skull was examined in three separate numerical experiments. First, the values of sound speed, density, and absorption assigned to the skull layer were separately perturbed by a linear variation. The maximum variation assigned to sound speed and density was $\pm 60 \%$ based on the range of acoustic properties reported in the IT'IS material properties database (see Fig. 2). A wider range of $\pm 90 \%$ was tested for absorption, due to the difficulty in accurately measuring tissue absorption [31]. Acoustic properties were varied in steps of $1 \%$ for the range $\pm 10 \%$, and in steps of $2 \%$ beyond that.

Second, the acoustic medium properties were individually perturbed by Gaussian noise with a coefficient of variation ranging from $1 \%$ to $20 \%$ in steps of $1 \%$. The average property value assigned to the skull layer was kept constant. It should be noted that this noise was applied at the grid resolution (see Fig. 3(b)), and as such does not correspond to realistic heterogeneities in skull bone. Instead it represents an uncertainty in the assigned medium properties, such as the impact of noise in pixel intensity when properties are derived from medical images. Sound speed and density values were constrained between the minimum and maximum values for cortical and trabecular bone reported in the IT'IS tissue property database [32] (See Fig. 2). Absorption was constrained between zero and three times the reference calculated using the power law parameters outlined in Section 2.1 .

Third, a multi-dimensional sensitivity analysis was performed, with variations in $\mathrm{HU}$ used to generate coupled changes in density, sound speed, and absorption based on the Aubry et al. 

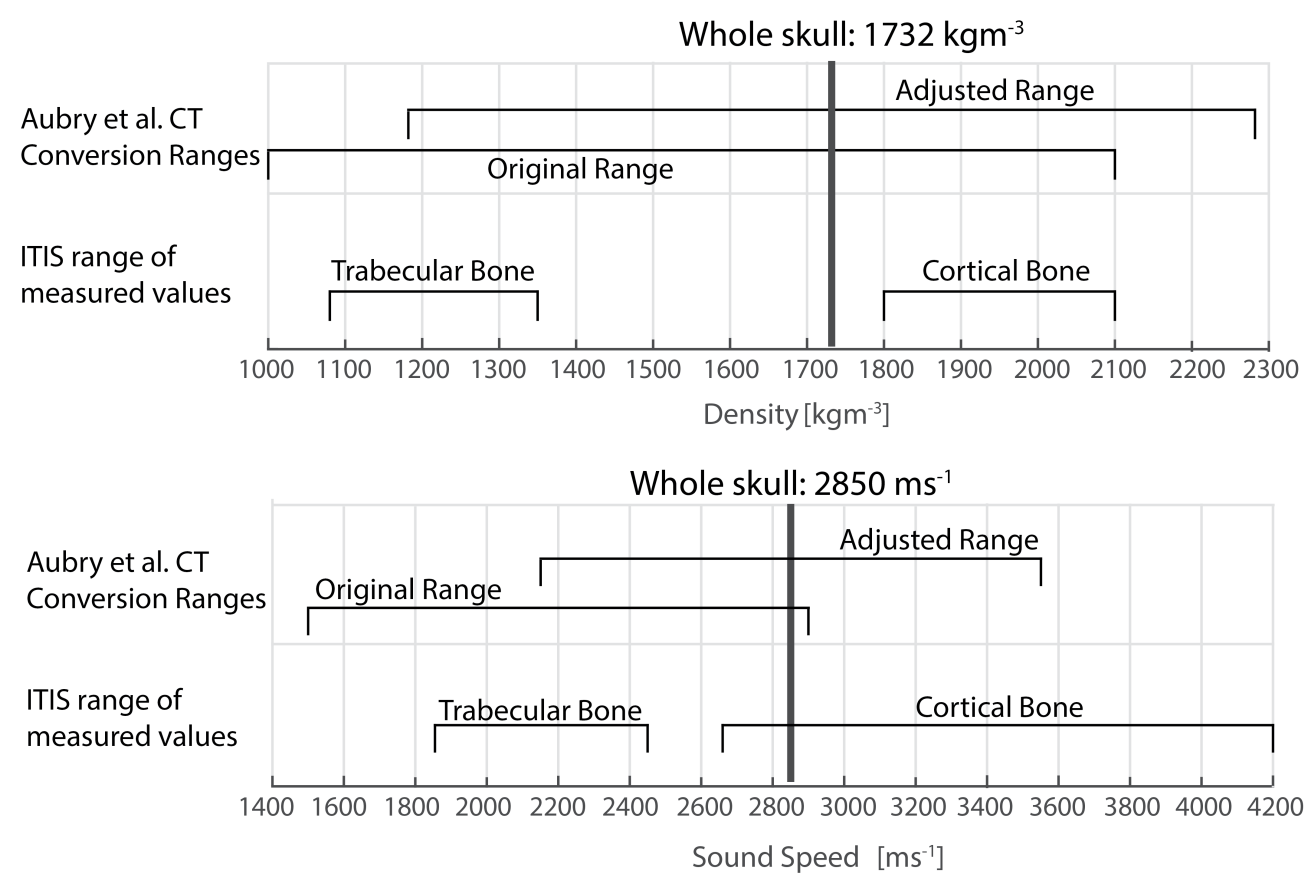

Figure 2: Whole skull values of sound speed and density used as reference values in the sensitivity analysis (taken from White et al. [7]). The range of measured values for bone taken from the IT'IS database [32] are shown for comparison. The original range of sound speed and density values used in the Aubry et al. CT conversion algorithm [5], and the adjusted values which yield the reference whole skull values for an HU value of 500, are also shown.

conversion algorithm [5]. This algorithm uses CT image HU, normalised between water (0 HU) and cortical bone (1000 HU), to derive a map of porosity:

$$
\phi=1-(\mathrm{HU} / 1000)
$$

This is then used to interpolate linearly between reference values for density $\left(\rho_{\min }, \rho_{\max }\right)$ and sound speed $\left(c_{\min }, c_{\max }\right)$ in water and bone

$$
\begin{aligned}
& \rho=\phi \rho_{\min }+(1-\phi) \rho_{\max } \\
& c=c_{\min }+\left(c_{\max }-c_{\min }\right)(1-\phi)
\end{aligned}
$$

Porosity is also used to calculate acoustic absorption. However, a power law is used instead of linear interpolation, and high porosity results in an increase in the simulated absorption value. The expression for absorption is given by

$$
\alpha=\alpha_{\min }+\left(\alpha_{\max }-\alpha_{\min }\right) \phi^{\beta}
$$

where $\alpha_{\max }$ and $\alpha_{\min }$ are maximum and minimum reference values for absorption. Here, the minimum and maximum values for each parameter were chosen such that Eqs. 1-4 give the reference whole skull properties outlined in Sec. 2.1 for an HU value of 500 (see Fig. 2). The value of HU was then perturbed to give coupled changes in density, sound speed, and absorption. Previous studies 

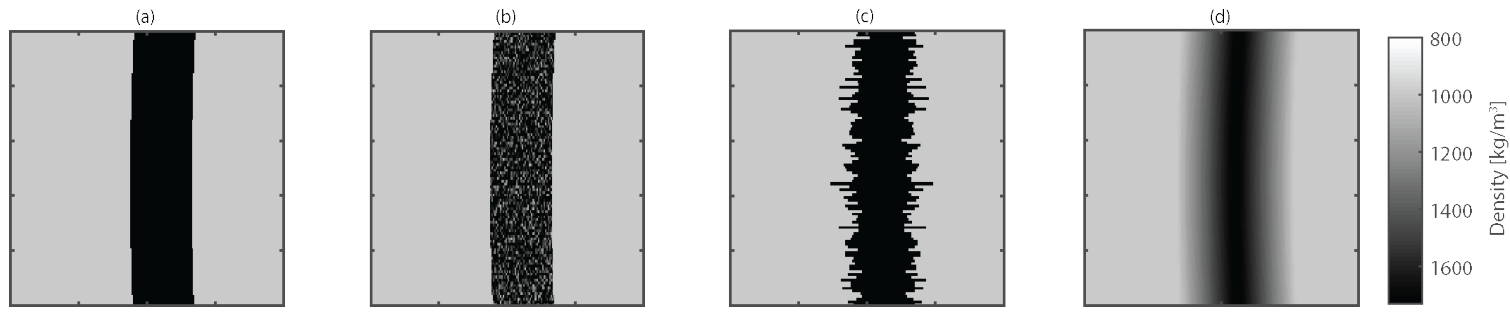

Figure 3: Selected density maps used in sensitivity analysis. (a) Reference bone layer. (b) Noisy variation in the medium properties. (c) Random variation in the skull thickness. (d) Smoothing of the bone layer.

into variation in CT $\mathrm{HU}$ have described up to $25 \% \mathrm{HU}$ error for densities of over $1100 \mathrm{~kg} \mathrm{~m}^{-3}$ [33], dependent on the calibration. Consequently, a range of $\pm 250 \mathrm{HU}$, in steps of $2 \mathrm{HU}$ up to $42 \mathrm{HU}$, and steps of $4 \mathrm{HU}$ from there, was tested.

\subsection{Sensitivity to the Geometry of the Skull}

The sensitivity of the intracranial field to changes in the geometry of the bone layer was examined in three separate numerical experiments. First, the effect of systematic changes in the bone thickness, which can occur when segmenting CT or MRI images [16], was examined by increasing and decreasing the thickness of the bone layer up to $\pm 3.9 \mathrm{~mm}$ in steps of $0.15 \mathrm{~mm}$. Second, the distribution of thickness across the length of the bone layer was varied randomly according to a Gaussian distribution with an increasing coefficient of variance, in steps of $1 \%$ up to a maximum of $20 \%$. This was to examine the potential influence of uncertainties in the exact position of boundaries on transcranial propagation, as shown in Fig. 3(c). The random thickness of the bone layer was constrained between 1-12 mm based on previously reported maximum and minimum values for skull thickness [23]. Third, the potential impact of CT partial volume effects was examined via spatial smoothing of the skull layer. Smoothing was achieved by applying a square smoothing kernel of increasing size, in steps of $0.15 \mathrm{~mm}$ up to a maximum of $5.85 \mathrm{~mm}$, to maps of acoustic properties, as a simulacrum of CT pixel size. An example is shown in Fig. 3(d).

\subsection{Experimental Validation}

Sonication experiments were carried out to corroborate the results of the numerical sensitivity analysis. The pressure field generated by a $1 \mathrm{MHz}$ focused transducer was measured following transmission through one of two skull bone phantoms developed with computer-aided design (CAD), described in Appendix A. This enabled the translation of the experiment into a registered k-Wave simulation. Comparison of the experimental and simulated fields was then used to evaluate the impact of uncertainties in the modelled sound speed and attenuation (modelled as absorption).

The first set of sonication experiments used a phantom designed with the same shape as the bone layer from the numerical sensitivity analysis. This will be referred to as the "parametric" phantom and was cast in Araldite 1302 resin, chosen due to the proximity of its reported acoustic properties to the skull values shown in Fig. 2 [34]. To obtain the maximum verisimilitude in registered simulations, these properties were measured, as described in Appendix B. The second phantom was created from the posterior portion of a mesh segmented from MRI head image data. This will be referred to as the "mesh-based" phantom, and was created in two materials, VeroBlack 3D printing material [35] and Araldite 1302. The reported and measured property values, and estimated uncertainty in those measurements, are shown in Table 2. 


\begin{tabular}{ccccc}
\hline & Comp. Speed $\left[\mathrm{m} \mathrm{s}^{-1}\right]$ & Shear Speed $\left[\mathrm{m} \mathrm{s}^{-1}\right]$ & Absorption $\left[\mathrm{dB} \mathrm{cm}^{-1}\right]$ & Density $\left[\mathrm{kg} \mathrm{m}^{-3}\right]$ \\
\hline Araldite 1302 & $3008 \pm 220$ & $1562 \pm 30$ & $1.81 \pm 0.6$ & 1637 \\
VeroBlack & $2495 \pm 8$ & $1081 \pm 31$ & $3.70 \pm 0.1$ & 1180 \\
\hline
\end{tabular}

Table 2: Compressional \& shear speed, attenuation and density of Araldite 1302 resin and VeroBlack 3D printing material [35] including estimated/reported uncertainty.

(a)

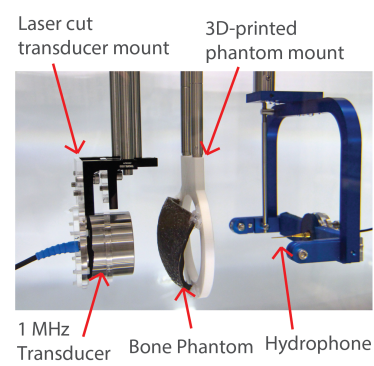

(b)

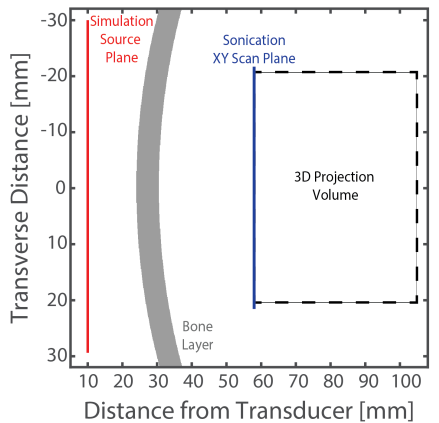

(c)

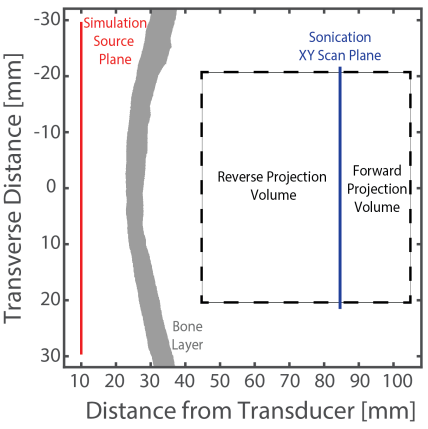

Figure 4: Sonication \& registered simulation layout. (a) Sonication setup for scanning following transmission through phantom. (b) Registered simulation setup for the parametric skull phantom. (c) Registered simulation setup for the anatomical skull phantom.

The ultrasound source used was a Precision Acoustics PZT HIFU transducer with a diameter of $60 \mathrm{~mm}$ and a focal length of $75 \mathrm{~mm}$. The transducer was driven at its nominal centre frequency of 1 $\mathrm{MHz}$ with a 3 cycle toneburst, generated by an Agilent 33522A Arbitrary Waveform Generator (Agilent, Berkshire, UK) and amplified by an E\&I A075 RF power amplifier (Electronics \& Innovation Ltd., Rochester, NY). The drive signal was monitored by a Tektronix DPO-5034B oscilloscope (Tektronix Inc., Beaverton, OR), also used to process hydrophone measurements. Measurements were carried out in a bath of degassed and deionised water with an integrated three-axis positioning system (Precision Acoustics, Dorchester, UK). Signals were acquired with a $0.2 \mathrm{~mm}$ needle hydrophone, connected to the oscilloscope via a submersible preamplifier and DC coupler (Precision Acoustics, as above). Water temperature was monitored continuously with a thermocouple (National Instruments Corporation, Austin,TX). The experimental setup with a resin phantom in place is shown in Fig. 4 (a).

To examine the experimental peak pressure field, the time varying pressure was recorded across a plane following transmission through the phantom. These planes were positioned at $58 \mathrm{~mm}$ and $85 \mathrm{~mm}$ from the transducer surface for the parametric and mesh-based phantoms, respectively. The time-varying pressure plane was then bandpass filtered, and projected forward and (in the case of the mesh based phantom) backward using linear acoustic holography to establish the pressure field across a volume encompassing the transducer focus. The position of the scan planes and 3D volumes are shown in Fig. 4. The time varying pressure was also recorded across a plane $45 \mathrm{~mm}$ from the centre of the transducer without the phantoms in place, to establish the source conditions for simulation.

Simulations designed to mimic the experimental sonications were then performed. The source conditions were calculated by time-reversing the measured free-field pressure signals and 
(a)

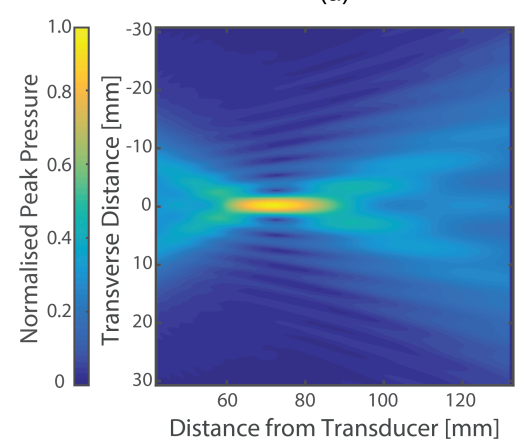

(b)

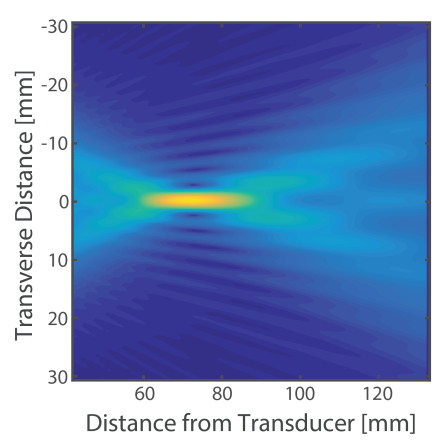

(c)

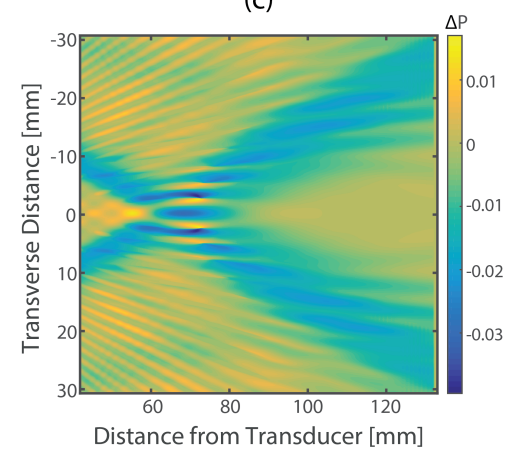

Figure 5: Results of elastic vs fluid comparison at $750 \mathrm{kHz}$ showing the steady state maximum pressure. (a) Fluid simulation. (b) Elastic simulation. (c) Difference plot.

backprojecting them to a plane $10 \mathrm{~mm}$ from the transducer surface. To create the map of acoustic medium properties, the parametric phantom was incorporated into the simulation based on a priori knowledge of its 3D shape and position. For the mesh based phantom, the 3D mesh was directly converted into a binary map of medium properties. The background was assigned the properties of water based on the recorded temperature of the water in the tank. To examine the accuracy of the registered simulations with reference to the experimental sonications, the maximum pressure was recorded across a volume matching the 3D projection volumes shown in Fig. 4. For both experimental and simulated volumes, the time varying pressure was also extracted at the position of the peak pressure in the experiment.

\section{Results}

\subsection{Effect of Shear Waves in the Skull}

The change in the peak pressure field due to including or excluding shear waves in the bone layer for a source frequency of $750 \mathrm{kHz}$ is shown in Fig. 5. The maximum change in peak intracranial pressure was $+0.09 \%$ for $500 \mathrm{kHz},-2.81 \%$ for $750 \mathrm{kHz}$, and $-0.80 \%$ for $1 \mathrm{MHz}$, respectively. Changes in the position of the centre of mass of the $-6 \mathrm{~dB}$ focus area all occurred along the beam axis, and were all less than $0.3 \mathrm{~mm}$. The change in the $-6 \mathrm{~dB}$ focal area was less than $1.5 \%$ for all frequencies. An L2 error norm was also calculated across the peak pressure field between elastic and fluid simulations for each frequency. The maximum L2 error was $4.94 \%$ for the $750 \mathrm{kHz}$ frequency, which also demonstrated the maximum error in peak pressure amplitude and position. L2 errors were $3.51 \%$ and $1.77 \%$ for $500 \mathrm{kHz}$ and $1 \mathrm{MHz}$ ultrasound, respectively. These results serve as a justification for neglecting shear in the 3D numerical sensitivity analysis. However, it should be noted that the limited impact of shear mode propagation may not hold for geometries involving more oblique angles of incidence. During testing it was noted that the changes in the field caused by the introduction of shear mode propagation begin to increase in magnitude once the shear speed in the bone layer was greater than $1800 \mathrm{~m} \mathrm{~s}^{-1}$. The effect of shear absorption was negligible. 

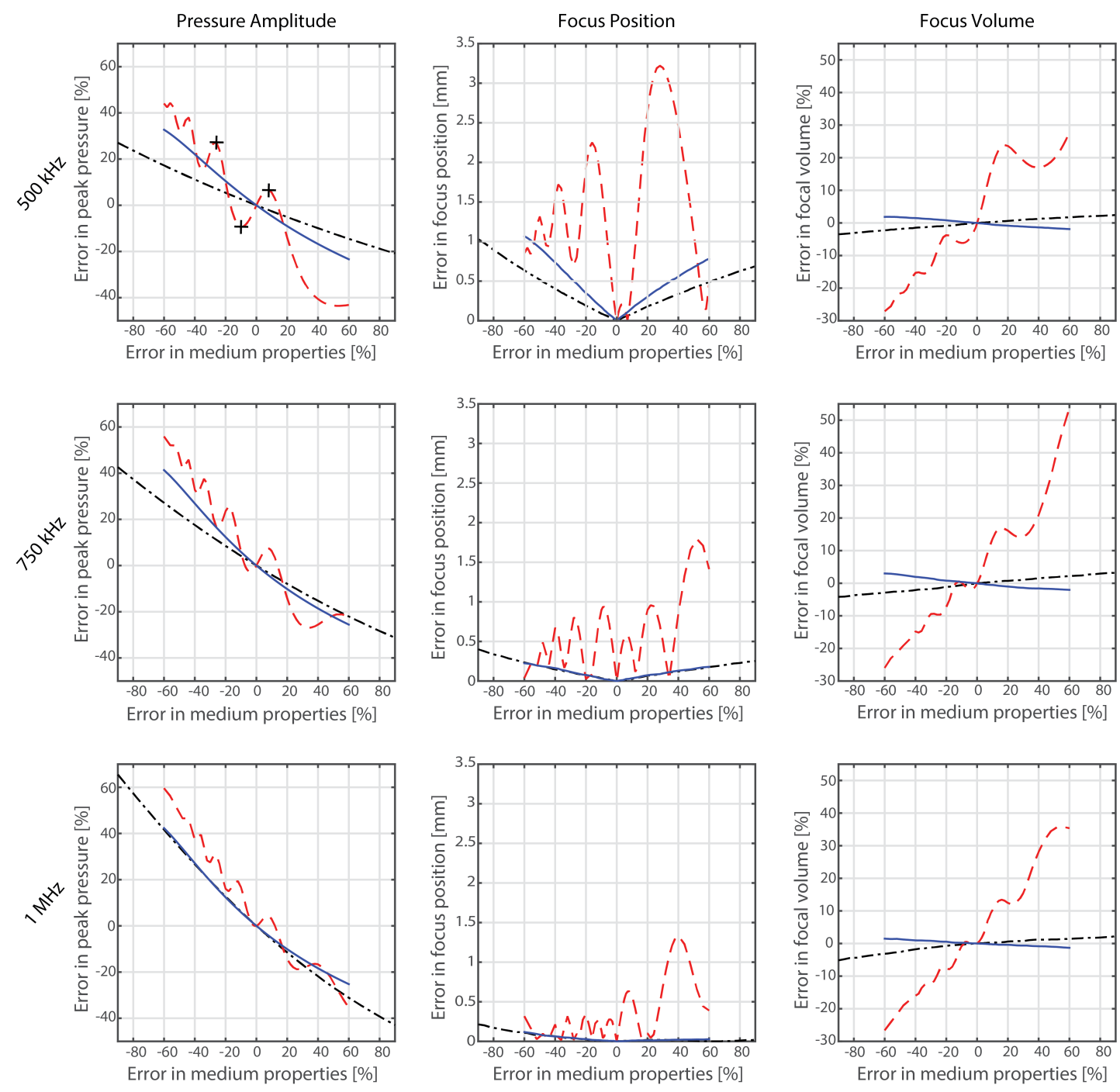

Figure 6: Errors in the intracranial field resulting from a linear variation of the acoustic medium properties. Rows correspond to frequencies, columns to the error metrics evaluated. Points marked on the $500 \mathrm{kHz}$ pressure amplitude plot correspond to the field plots shown in Fig. 7 .

\subsection{Sensitivity to Linear Variation in the Acoustic Properties of the Skull}

The errors in the intracranial field resulting from linear variation in acoustic properties are shown in Fig. 6. Several conclusions can be drawn from these results. Overestimation of the sound speed, density and absorption all lead to a corresponding decrease in the simulated transcranial pressure, and vice versa. This is due, at least in part, to the increased acoustic impedance (a product of sound speed and density) and absorption, resulting in increased attenuation. To obtain an error in peak 
(a)

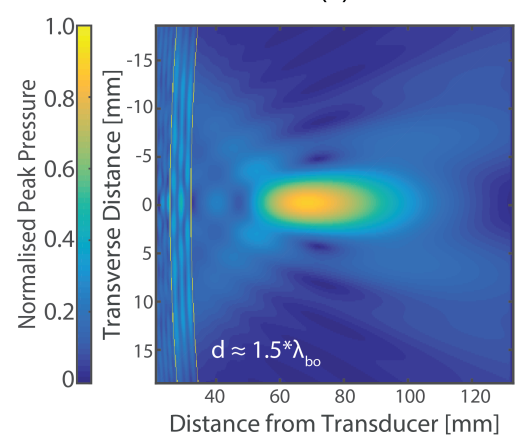

(b)

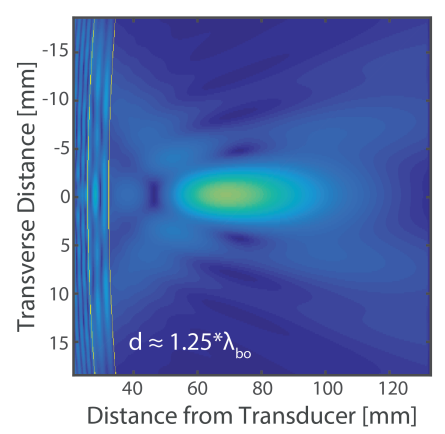

(c)

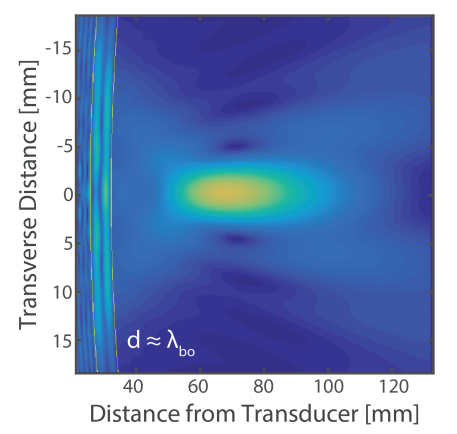

Figure 7: Slices through the $500 \mathrm{kHz}$ peak pressure field at increasing sound speeds, where d is the thickness of the bone and $\lambda_{\text {bo }}$ is the acoustic wavelength. (a) Field when sound speed in the bone layer is $2109 \mathrm{~m} / \mathrm{s}$ $\left(\mathrm{d} \approx 1.5 \lambda_{\mathrm{bo}}\right)$. (b) Field when the sound speed is $2565 \mathrm{~m} / \mathrm{s}\left(\mathrm{d} \approx 1.25 \lambda_{\mathrm{bo}}\right)$. (c) Field when the sound speed is $3050 \mathrm{~m} / \mathrm{s}\left(\mathrm{d} \approx \lambda_{\mathrm{bo}}\right)$.

pressure of less than $5 \%$ for all frequencies, sound speed and density must be assigned with a change of less than $4 \%$ and $9 \%$, respectively. To obtain less than a $5 \%$ error in peak pressure, absorption must be assigned with a change of less than $9 \%$ at $1 \mathrm{MHz}$, increasing to $20 \%$ at $500 \mathrm{kHz}$. This is due to the increase in absorption with frequency.

The position and volume of the focus also depend on all three acoustic properties. However, changes in density and absorption do not cause errors of larger than $1.5 \mathrm{~mm}$ in position or $5 \%$ in focal volume. To obtain less than $20 \%$ error in focal volume across all frequencies, sound speed must be defined with less than $11 \%$ variation. To obtain an error in focus position of less than $1.5 \mathrm{~mm}$ at $500 \mathrm{kHz}$, sound speed must be defined with a variation of less than $9 \%$. As the sound speed changes further, the position of the focus can deviate up to $3 \mathrm{~mm}$ from the reference position, but does not vary steadily. The focus position oscillates around a fixed point for all frequencies, with the amplitude of the oscillation and the dependence on sound speed informed by the ultrasonic frequency. Error in focus position is highest at $500 \mathrm{kHz}$, due to the longer wavelength and the distance of the reference focus from the fixed point around which the focus oscillates.

Errors in peak pressure and focus volume also oscillate with changing sound speed. An example of the oscillating peak pressure is shown in Fig. 7, with the bone layer highlighted. These plots correspond to maxima and minima in the oscillating pressure pattern (see Fig. 6), and occur when the thickness of the bone layer corresponds to particular multiples of the wavelength of $500 \mathrm{kHz}$ ultrasound in the layer. This relationship to wavelength does not hold for the oscillations in focus position in the same way, which is likely due to the lensing behaviour of the bone layer changing with sound speed instead.

These results show that, for the skull geometry simulated, small systematic variation in the assigned medium properties can result in significant changes in the size and position of the focus. They also indicate that accurate assignation of sound speed is of primary importance when modelling transcranial propagation. Changes in the assigned absorption and density can lead to comparable errors to sound speed in the simulated peak intracranial pressure. 

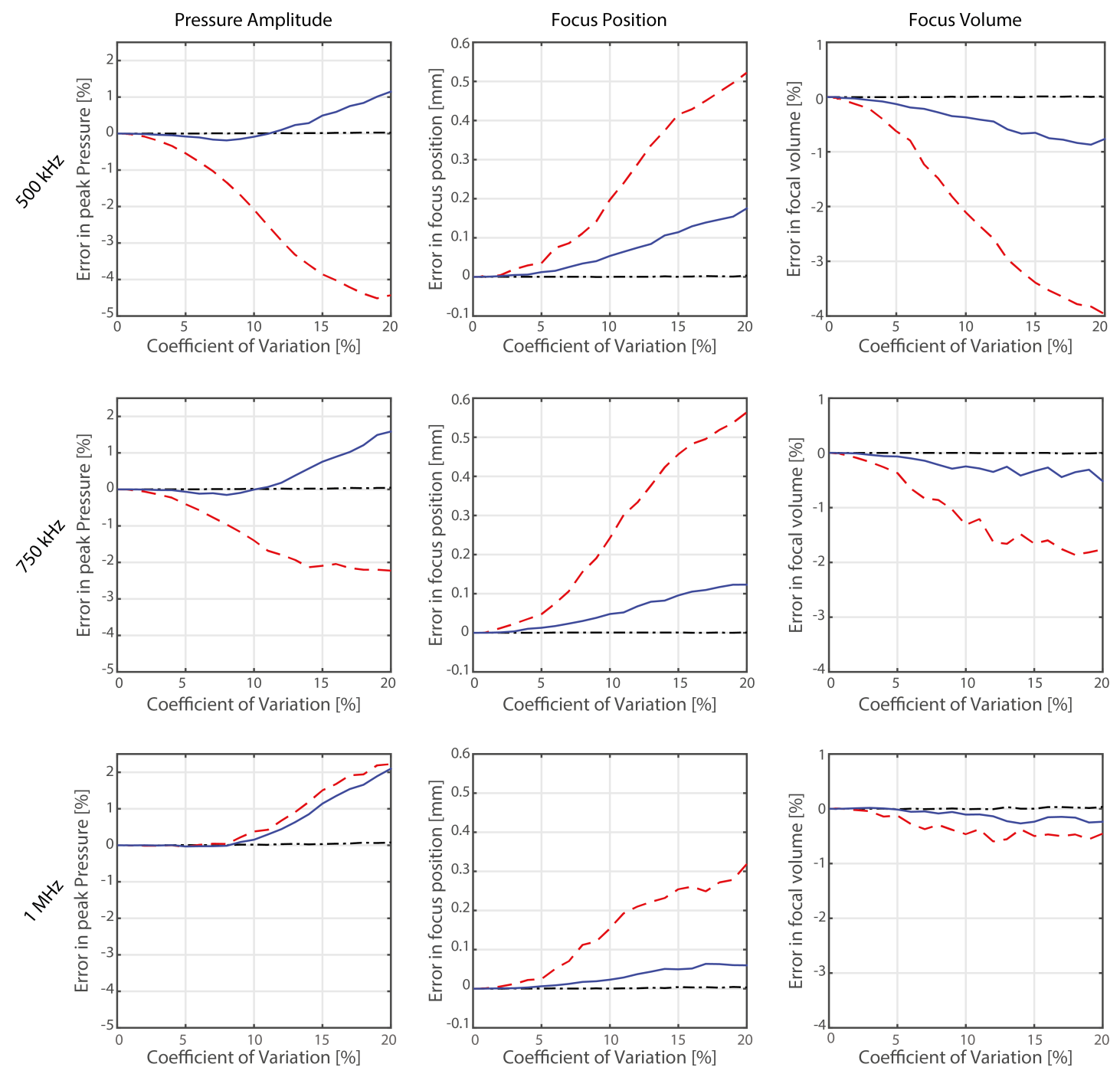

Legend: Sound Speed - - Density —

Figure 8: Errors in field resulting from noisy perturbation of acoustic properties with an increasing coefficient of variation. Rows correspond to frequency tested and columns correspond to error metric evaluated.

\subsection{Sensitivity to Noisy Variation in the Acoustic Properties of the Skull}

The errors in the intracranial field resulting from noisy perturbation of the acoustic properties of the skull layer are shown in Fig. 8. Several conclusions can be drawn from these results. First, the errors in the field resulting from noisy variation in medium properties are smaller than those from linear changes. Errors in peak pressure and focus volume remain below 5\%, while the error in focus position remains below $1 \mathrm{~mm}$. Second, the introduction of noise into the map of acoustic absorption (while keeping the average absorption constant) has almost no effect on the intracranial 
(a)

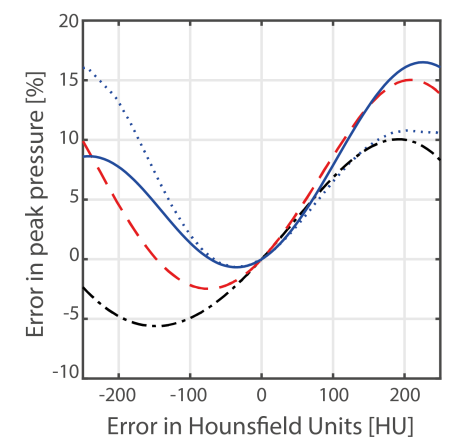

(b)

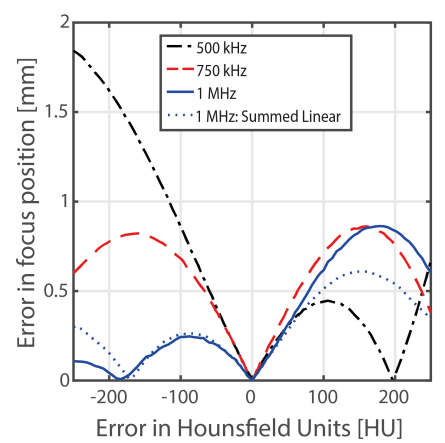

(c)

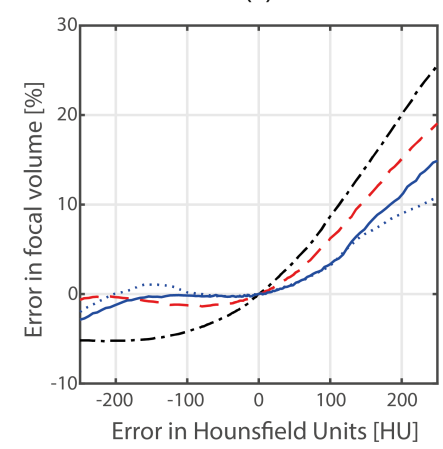

Figure 9: Errors in the intracranial field resulting from variation in Hounsfield Units used to derive the bone layer medium properties, and a comparison with the sum of linear changes shown in Fig. 6 for $1 \mathrm{MHz}$. (a) Percentage error in peak pressure. (b) Error in focus position. (c) Percentage error in focal volume.

field. This would appear to vindicate previous studies where a homogeneous map of absorption has been used alongside fully heterogeneous maps of sound speed and density [12]. Finally, errors in the intracranial field are generally less severe at higher frequencies. This is most apparent for the error in focus volume, but can also be observed across the other error metrics.

\subsection{Sensitivity to Variation in CT Hounsfield Units}

The errors in the intracranial field resulting from variation in the CT HU used to derive the acoustic properties are shown in Fig. 9. Across the range of HU tested, the maximum error in peak pressure was an increase of $17 \%$ for $1 \mathrm{MHz}$ ultrasound. To obtain less than $5 \%$ error in peak pressure, a change of less than $60 \mathrm{HU}$ is required. For $500 \mathrm{kHz}$, a variation of no greater than $180 \mathrm{HU}$ results in a positional error of less than $1.5 \mathrm{~mm}$, while the error remains below $1 \mathrm{~mm}$ for higher frequencies. The focal volume increases at higher HU values, with a variation of $200 \mathrm{HU}$ causing an error in focal volume of $20 \%$, while reducing $\mathrm{HU}$ appears to have a lesser effect on focal volume. Errors in the intracranial field oscillate, in line with the linear changes in sound speed shown in Fig. 6 .

Within the range of $\mathrm{HU}$ tested, the maximum errors across all metrics are lower than the errors shown in Fig. 6 for individual changes in assigned medium properties. This suggests that the primary concern should be the robustness of any method for the conversion of CT images to density, absorption and especially sound speed, with the accuracy of HU measurements being a secondary concern. Figure 9 also compares the error resulting from the sum of equivalent individual linear errors (from Fig. 6) for $1 \mathrm{MHz}$ ultrasound. While they match well for low $\mathrm{HU}$ variation, when reducing $\mathrm{HU}$ the sum of linear errors is less than the error resulting from the simultaneous alteration of all properties, and vice versa.

\subsection{Sensitivity to Linear Variation in the Skull Thickness}

The errors in the intracranial field resulting from linear variations in bone layer thickness are shown in Fig. 10. A change in layer thickness of $0.1 \mathrm{~mm}$ is sufficient to cause a peak pressure error of $5 \%$ for all frequencies, while a change in skull thickness of $\pm 1 \mathrm{~mm}$ can lead to an error of up to $\pm 20 \%$, depending on frequency. Beyond this level, underestimation of the layer thickness has a greater 
(a)

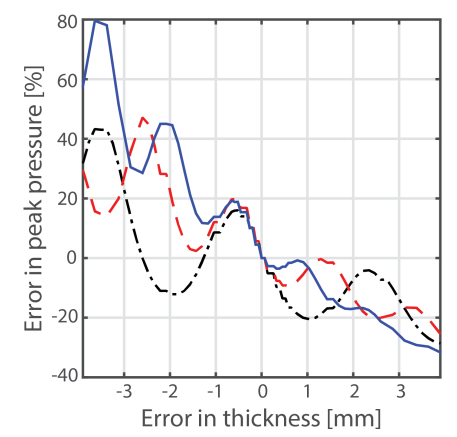

(b)

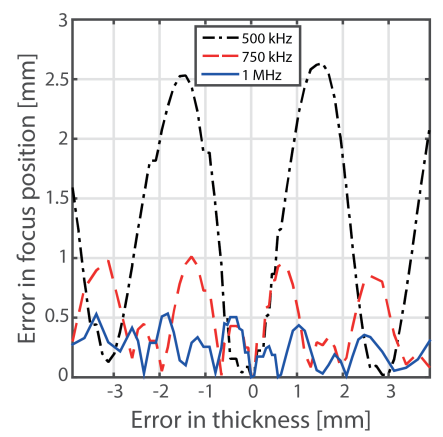

(c)

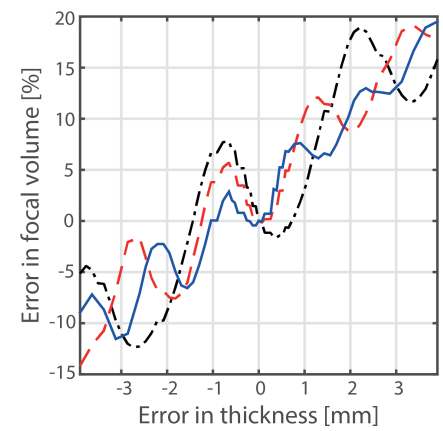

Figure 10: Errors in field resulting from systematic over/under estimation of the bone layer thickness, for a reference thickness of $6.5 \mathrm{~mm}$. (a) Percentage error in peak intracranial pressure. (b) Error in the position of the focus. (c) Percentage error in focus volume.

effect on the peak intracranial pressure than overestimation. The position of the focus changes with thickness up to maximum errors of $2.5 \mathrm{~mm}, 1 \mathrm{~mm}$, and $0.5 \mathrm{~mm}$ for $500 \mathrm{kHz}, 750 \mathrm{kHz}$, and $1 \mathrm{MHz}$, respectively. A change in thickness of less than $0.8 \mathrm{~mm}$ is required to obtain a positional error of less than $1.5 \mathrm{~mm}$ for $500 \mathrm{kHz}$. The error in focal volume shows a general trend of increasing with larger layer thickness and vice versa, but did not exceed $\pm 20 \%$ across the range of thicknesses tested.

Oscillations in the error metrics can be observed, similar to the oscillations seen for changes in sound speed in Fig. 6. These are related to wavelength, as can be seen by the relative periods of the oscillations in Fig. 10 (b). This supports previous conjecture regarding the influence of the wavelength of applied ultrasound relative to the thickness of the layer, and the lensing effects of the layer.

\subsection{Sensitivity to Random Variation in the Skull Thickness}

The errors in the intracranial field resulting from random perturbations to the skull layer thickness are shown in Fig. 11. Introducing a noisy variation in the thickness can have a large effect on the peak intracranial pressure, with an overestimation of peak pressure with increasing perturbation of the thickness. Coefficients of variation in thickness of $1 \%, 2 \%$ and $3 \%$ cause an error in peak pressure of $5 \%$ for $500 \mathrm{kHz}, 750 \mathrm{kHz}$ and $1 \mathrm{MHz}$, respectively, rising to a maximum error of around $50 \%$. It is not clear why this should be the case, as scattering could be expected to reduce focused transmission. It is possible that the random scattering reduces existing destructive interference effects, or that the random perturbation alters the scattering profile to increase the forward transmission of ultrasound. Regardless, these results do suggest that uncertainty in the profile of the bone layer, and modelling the layer as closer to or further from a smooth layer of even thickness, can alter the predicted peak intracranial pressure. The error in focus position was below $0.5 \mathrm{~mm}$ for $1 \mathrm{MHz}$ and $750 \mathrm{kHz}$, but increased to over $1.4 \mathrm{~mm}$ for $500 \mathrm{kHz}$ ultrasound. The increased impact at higher frequencies is likely due to the larger scale of the changes in thickness relative to the wavelength of the ultrasound. The error in focal volume is low, not rising above $3 \%$ across the frequencies and range of errors tested. 
(a)

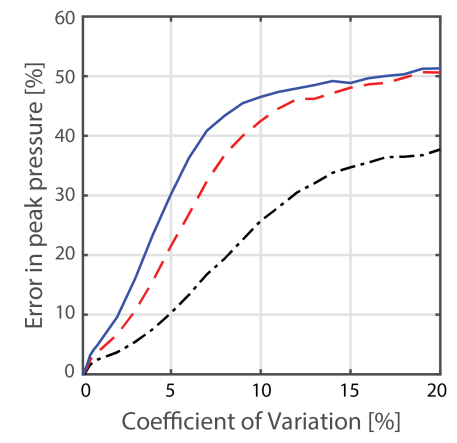

(b)

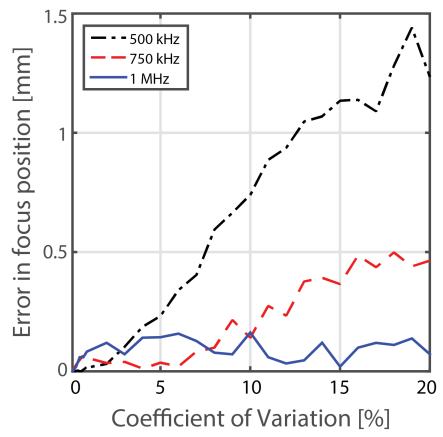

(c)

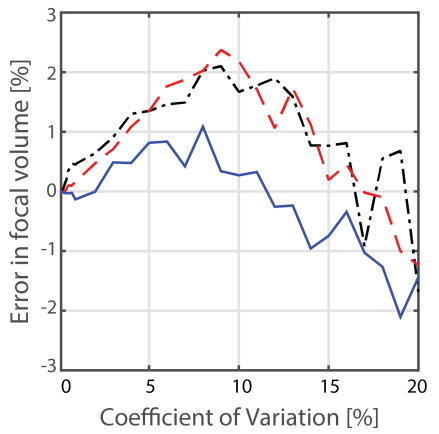

Figure 11: Errors in field resulting from noisy perturbation of the bone layer thickness at multiple frequencies. (a) Percentage error in peak pressure. (b) Error in focus position. (c) Percentage error in focal volume.

(a)

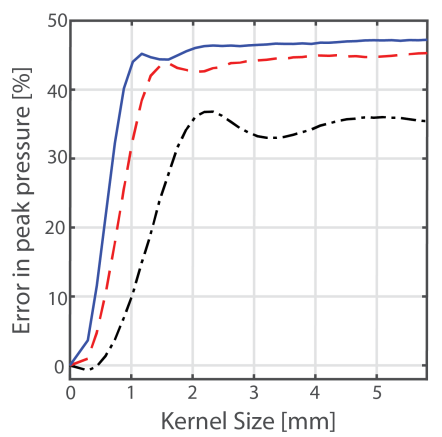

(b)

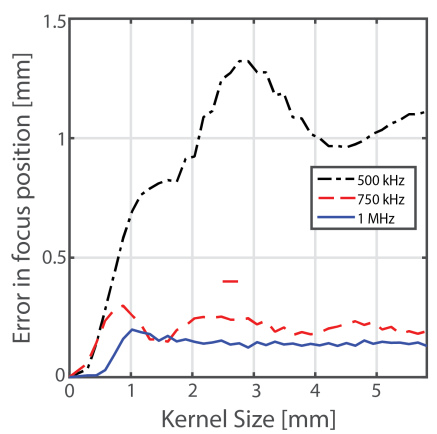

(c)

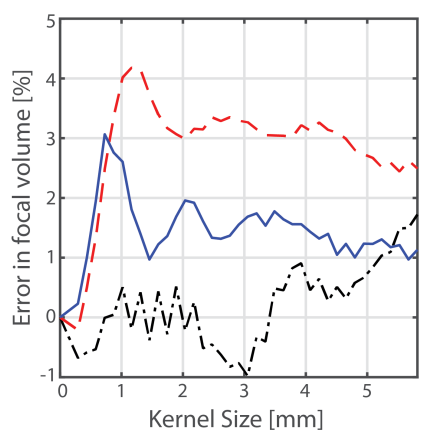

Figure 12: Errors in field resulting from kernel smoothing of the bone layer at multiple frequencies. a) Percentage error in peak pressure. (b) Error in focus position. (c) Percentage error in focal volume.

\subsection{Sensitivity to Smoothing of the Bone Layer}

The errors in the intracranial field resulting from smoothing the map of medium properties are shown in Fig. 11. There is a clear overestimation of transcranial transmission dependent on the level of smoothing and the frequency of the applied ultrasound, with higher frequencies more affected. Kernels of $0.35 \mathrm{~mm}, 0.45 \mathrm{~mm}$, and $0.8 \mathrm{~mm}$ cause an error in peak pressure of greater than $5 \%$ for 500 $\mathrm{kHz}, 750 \mathrm{kHz}$, and $1 \mathrm{MHz}$ ultrasound, respectively. For a kernel size of $1 \mathrm{~mm}^{3}$ the peak transcranial pressure is increased by over $50 \%$ for $1 \mathrm{MHz}$ ultrasound. Given that the scale of modern CT imaging systems falls well within this range, this implies that partial volume smoothing of CT images could potentially have a dramatic effect on simulated transcranial fields if the smoothing of the CT images is translated into the map of acoustic medium properties. There is some error in the position of the focus with increasing smoothing, although it only rises above $1 \mathrm{~mm}$ for $500 \mathrm{kHz}$ ultrasound. The error in the focal volume is small, never rising above $5 \%$. 
(a)

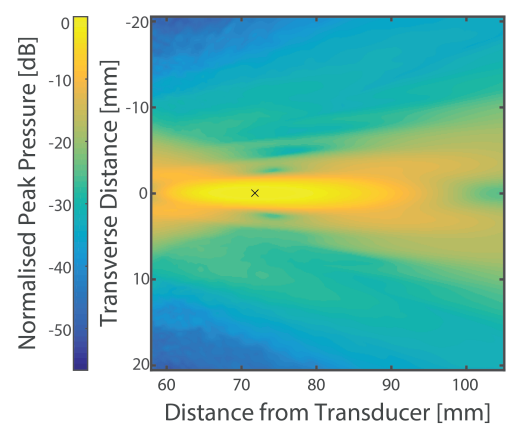

(b)

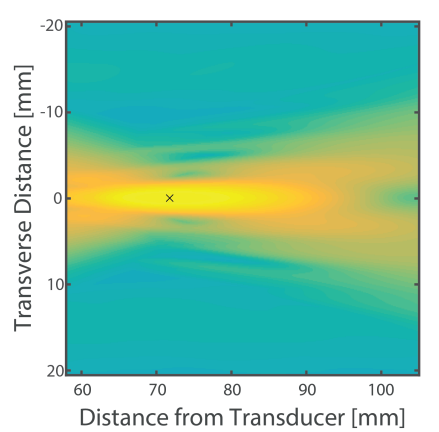

(c)

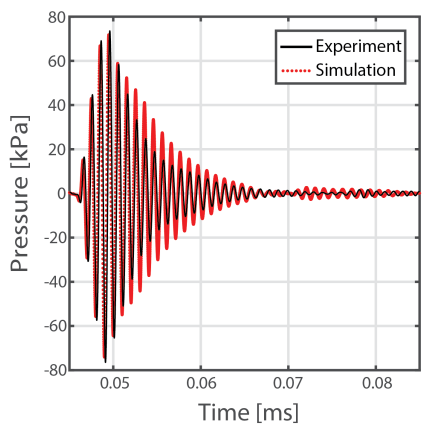

Figure 13: Results of experiment and simulation through parametric phantoms. (a) Experimental peak positive pressure measured on the axial-lateral plane. (b) Simulated peak positive pressure on the axiallateral plane. (c) Comparison of time varying pressures at the measured spatial peak pressure (marked by an $\mathrm{X}$ ).

\subsection{Experimental Validation using a Parametric Skull Phantom}

A comparison of the experimental and simulated fields for the parametric skull phantom is shown in Fig. 13. The peak pressure in the simulation relative to the experiment was underestimated by $2.1 \%$, the focus position moved by $0.137 \mathrm{~mm}$, and the simulated focal volume was $2.03 \%$ larger. When evaluated with reference to the uncertainty in sound speed and attenuation described in Appendix $\mathrm{B}$, and the results of the numerical sensitivity analysis, all metrics fall well within the expected tolerances.

The experimental and simulated time-varying pressure signals at the position indicated in the peak pressure fields is shown in Fig. 13(c). The registered simulation predicts a slightly earlier time of arrival for the waveform shown compared to the experiment. There is also a small difference in the pulse shape between experimental and simulated signals. The simulated pulse has a slightly lower peak amplitude, and a longer ring down. Based on this discrepancy, and the general trends shown in Fig. 6, it is probable that one or both of the attenuation and sound speed values assigned to the bone layer were too high. However, overall there is good quantitative agreement between the two signals.

\subsection{Experimental Validation using an Anatomical Skull Phantom}

The results of the experimental sonications and registered simulations through the mesh-based phantoms are shown in Fig. 14. Simulation of propagation through the Araldite mesh-based phantom underestimated the peak pressure by $0.8 \%$ compared to the experimental measurement, the focus position was moved by $0.61 \mathrm{~mm}$, and the focus volume was overestimated by $11.6 \%$. While the errors in both peak pressure and focus position remain within expected variations, the error in focal volume is greater than would be expected for the maximum uncertainty of $220 \mathrm{~m} \mathrm{~s}^{-1}$. Furthermore, based on the results of the numerical sensitivity analysis, this error in focal volume should be accompanied by a correspondingly large change in, at least, peak pressure.

The simulation of propagation through the VeroBlack mesh based phantom underestimated peak pressure by $0.8 \%$, the focus position was moved by $0.87 \mathrm{~mm}$, and the focus volume was underestimated by $2.1 \%$. The closer agreement is likely due to more accurate medium property measurements. Figure 14 also shows the experimental and simulated time-varying pressure at the 
(a)

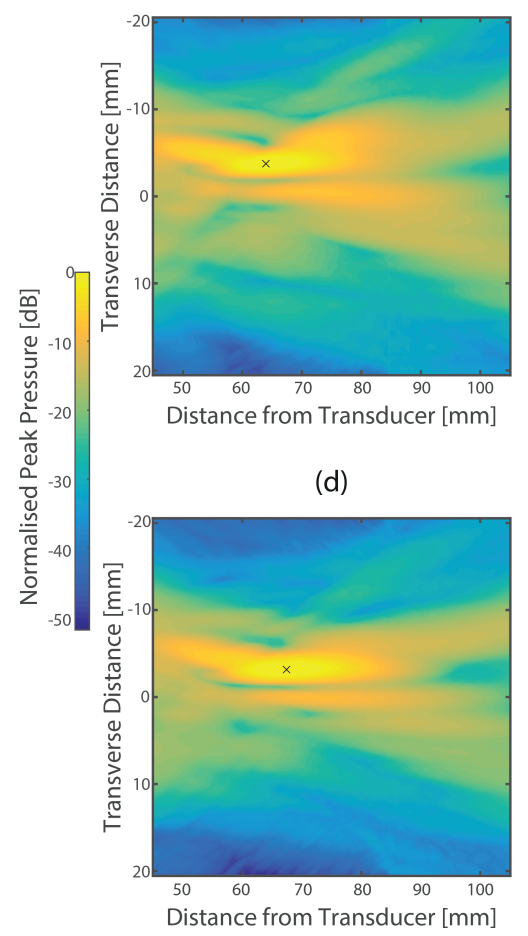

(b)

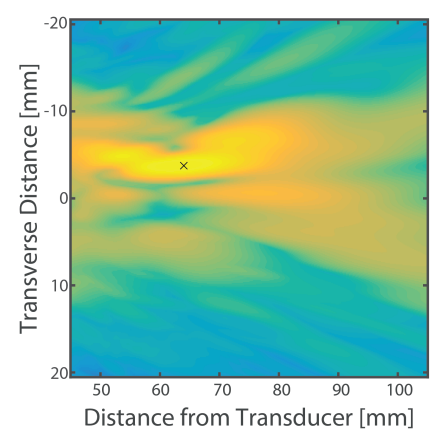

(e)

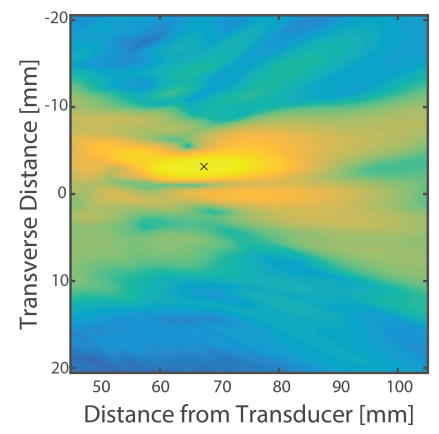

(c)

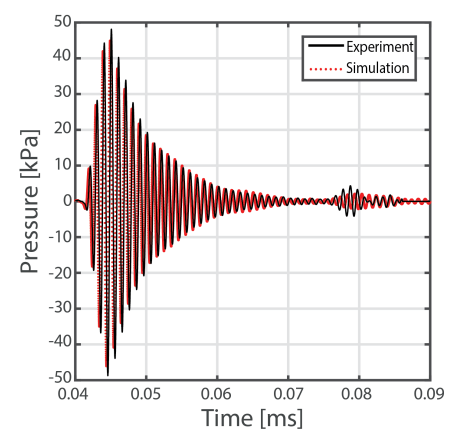

(f)

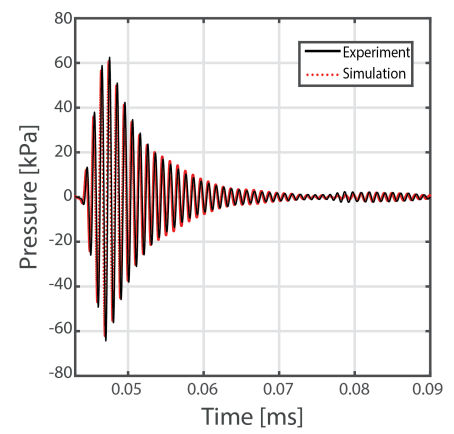

Figure 14: Results of experiments and simulations through mesh-based phantoms. The top row shows the results for the Araldite phantom, and the bottom row shows the results for the VeroBlack phantom. (a) \& (d) Experimental peak positive pressure measured across the axial-lateral plane. (b) \& (e) Simulated peak positive pressure across the axial-lateral plane. (c) \& (f) Comparison of the time-varying pressures at the measured peak pressure position (marked by an X).

peak pressure position in the experimental field. These results show good agreement in terms of pulse shape, amplitude and peak pressure, especially for the VeroBlack phantom.

The discrepancy in the results for the Araldite phantom could have a variety of causes. It is possible that the difference in medium geometry between the numerical sensitivity analysis and the mesh-based phantom has altered the sensitivity of the intracranial field to the phantom acoustic properties. However, the VeroBlack phantom has comparable errors in peak pressure and position without the larger error in focal volume. It is more likely that there is inaccuracy in the medium maps arising from the casting process, or errors in phantom alignment.

\section{Discussion and Conclusion}

The impact of the accurate mapping of acoustic medium properties on the intracranial field was examined via an idealised model of transcranial transmission. A sensitivity analysis was carried out, examining the impact of systematic changes and random uncertainty in the assigned medium properties, changes in the geometry of the layer, and smoothing of the bone layer. Before the sensitivity analysis, the impact of modelling shear mode propagation was examined, and determined to have a negligible impact for shear speed values of $<1800 \mathrm{~m} \mathrm{~s}^{-1}$. The results of the numerical 


\begin{tabular}{ccccccc}
\hline Frequency & $c[\%]$ & $\rho[\%]$ & $\alpha[\%]$ & HU & Thickness $[\mathrm{mm}]$ & Kernel $[\mathrm{mm}]$ \\
\hline $500 \mathrm{kHz}$ & $4 / 9 / 12$ & $10 / \mathrm{X} / \mathrm{X}$ & $18 / \mathrm{X} / \mathrm{X}$ & $71 / 150 / 200$ & $0.10 / 0.74 / \mathrm{X}$ & $0.35 / \mathrm{X} / \mathrm{X}$ \\
$750 \mathrm{kHz}$ & $4 / 46 / 39$ & $9 / \mathrm{X} / \mathrm{X}$ & $12 / \mathrm{X} / \mathrm{X}$ & $62 / \mathrm{X} / \mathrm{X}$ & $0.10 / \mathrm{X} / \mathrm{X}$ & $0.45 / \mathrm{X} / \mathrm{X}$ \\
$1000 \mathrm{kHz}$ & $4 / \mathrm{X} / 33$ & $8 / \mathrm{X} / \mathrm{X}$ & $7 / \mathrm{X} / \mathrm{X}$ & $71 / \mathrm{X} / \mathrm{X}$ & $0.12 / \mathrm{X} / \mathrm{X}$ & $0.80 / \mathrm{X} / \mathrm{X}$ \\
\hline
\end{tabular}

Table 3: Maximum permissible errors in the acoustic medium properties and geometry of the skull needed ensure less than $5 \%$ error in peak pressure / $1.5 \mathrm{~mm}$ in focus position / $20 \%$ error in focal volume. X indicates that these error criterion were not exceed for the range of variation tested.

sensitivity analysis are summarised in Table 3 .

In terms of the assignation of acoustic medium properties there are several relevant observations. The peak intracranial pressure is affected by the values of sound speed, density, and absorption. The impact is similar for each medium property, and the effect is larger at higher frequencies. The position and volume of the focus is most dramatically affected by changes in sound speed, although changes in density and absorption will have a minor effect. When altering sound speed, oscillations in each error metric were observed. This is due to the pattern of wavelength interference within the bone tissue layer, but is also influenced by changes in diffraction through the layer, and the resulting interference pattern in the intracranial field. To obtain less than $5 \%$ error in peak pressure, less than $1.5 \mathrm{~mm}$ error in focus position, and less than $20 \%$ error in focal volume, sound speed must be defined with less than $4 \%$ change, or $114 \mathrm{~m} \mathrm{~s}^{-1}$, and density must be defined with less than $9 \%$ change, or $156 \mathrm{~kg} \mathrm{~m}^{-3}$. Absorption has a significant effect on intracranial pressure only, and a change in the absorption coefficient of less than $9 \%$ is required to obtain less than $5 \%$ error in peak pressure for the highest frequency of $1 \mathrm{MHz}$. Random, noisy uncertainty in medium properties has a lesser effect on the intracranial field, and has almost no effect for uncertainty in absorption, indicating that the use of homogeneous absorption maps based on average absorption or transmission loss is justified. The impact of combined changes in medium assignment was also examined, with reference to a widely used algorithm for the conversion of $\mathrm{HU}$ to density, sound speed, and absorption. Across the range of typical uncertainty in $\mathrm{HU}$ tested, the overall errors in the intracranial field were less than observed for expected variations in individual medium properties. This strongly suggests that uncertainty in the $\mathrm{HU}$ of a $\mathrm{CT}$ image used to create a map of medium properties is less important than the robustness of the conversion algorithm.

For variation in medium geometry, systematic changes in layer thickness and smoothing on the scale of clinical CT resolution $(0.5-1 \mathrm{~mm})$ result in significant changes in simulated peak intracranial pressure. A systematic change in thickness of $0.1 \mathrm{~mm}$, or a smoothing kernel of $0.35-0.8 \mathrm{~mm}$ is sufficient to cause an error in peak pressure of $5 \%$. Focal volume and position are also affected, especially by systematic change in layer thickness. Noisy uncertainty in the layer thickness also affected peak intracranial pressure, with a coefficient of variation of $1 \%$ enough to increase the peak pressure by $5 \%$ for $1 \mathrm{MHz}$ ultrasound.

Consistently, the properties of the skull layer have a more significant effect on the peak pressure amplitude than on focus position or volume. This indicates that accurate maps of acoustic properties are likely to be less important for ablative applications, where fine control over the pressure is secondary to accurate targeting.

The results of the numerical sensitivity analysis were qualified through multiple registered experiments and simulations. A phantom based on the idealised map of the skull used in the numerical sensitivity analysis was created, and the field resulting from an experimental sonication through the phantom compared with a registered simulation of the same. The discrepancy in peak pressure amplitude and the size and position of the focus agreed well with the expected error based 
on the sensitivity analysis and the estimated uncertainty in the medium properties.

To place this work in context, Mueller et al. [18] examined the impact of changes in intracranial tissue models. They concluded that modelling different soft tissues separately was of limited importance, although large changes in brain tissue sound speed and density (scaling by a factor of two or more) did have a significant effect. They also observed that changing skull geometry from flat to curved reduced peak RMS intensity by $40 \%$, supporting the results of the present paper on the importance of geometry, and generally affirmed that the skull was the major material influence on the intracranial field.

A potential limitation of this work is the extent to which the results are generalisable. The sensitivity analysis was carried out with a single element transducer with no aberration correction, and a specific skull geometry designed to approximate the varied shaped of the skull. A homogeneous model with aggregate skull properties was used to simplify the introduction of different errors into the layer. The impact of variation in nonlinearity parameter was not examined, which may be relevant for high intensity applications. Different skull geometries may have different specific sensitivities to the errors examined in the numerical sensitivity analysis. Different transducer configurations may also influence the results. However, it is expected that the general scale of the resulting errors in the field, and the relative importance of different medium properties and aspects of medium geometry will be maintained. Moreover, as the transcranial field used in the sensitivity analysis was not strongly aberrated, the results should also extend to fields in which aberration correction is applied.

A second limitation is the inability of the sensitivity analysis to explain the discrepancy between experimental and simulated focal volume for propagation through the Araldite mesh-based phantom. It is possible that inadequacies in the casting process, or an error in stereotaxis or alignment, which were not examined in the sensitivity analysis, could have caused this. Despite these limitations, generally good agreement was observed between experiment and simulation of ultrasound propagation through phantoms. This was especially true for the VeroBlack simulations, likely due to the increased accuracy of measurements of its acoustic properties and a greater consistency in 3D printing compared to the mixing and casting process of Araldite.

The results presented here are of immediate relevance for applications that require accurate targeting of intracranial structures with ultrasound. They highlight the importance of accurate mapping of the bone layer, most notably the spatial accuracy of maps derived from CT images, and the sound speed values assigned to this map. These results also confirm the possibility of obtaining high accuracy in the position and size of the simulated focus, and the time varying pressure signals predicted at the focus when sufficiently accurate maps of medium properties are available. This will be important in optimising low-intensity applications of therapeutic ultrasound as they move towards targeting deep brain structures.

\section{Acknowledgements}

The authors would like to thank Eve Hatten and Daniil Nikitichev for advice and assistance with 3D printing, Nishant Ravikumar and Zeike Taylor for the provision of the skull and brain meshes, and Nader Saffari for the loan of a shear wave transducer. This work was supported by the Engineering and Physical Sciences Research Council (EPSRC), UK, grant numbers EP/L020262/1 and $\mathrm{EP} / \mathrm{M} 011119 / 1$. Computational resources were provided by the IT4Innovations Centre of Excellence project (CZ.1.05/1.1.00/02.0070), funded by the European Regional Development Fund and the national budget of the Czech Republic via the Research and Development for Innovations Operational Programme, as well as Czech Ministry of Education, Youth and Sports via the project Large Research, Development and Innovations Infrastructures (LM2011033). 
[1] K. D. Evans, B. Weiss, and M. Knopp, "High-Intensity Focused Ultrasound (HIFU) for Specific Therapeutic Treatments: A Literature Review," Journal of Diagnostic Medical Sonography, vol. 23, no. 6, pp. 319-327, 2007.

[2] R. J. Piper, M. A. Hughes, C. M. Moran, and J. Kandasamy, "Focused ultrasound as a non-invasive intervention for neurological disease: a review," British Journal of Neurosurgery, vol. 8697, pp. 1-8, apr 2016.

[3] W. Legon, T. F. Sato, A. Opitz, J. Mueller, A. Barbour, A. Williams, and W. J. Tyler, "Transcranial focused ultrasound modulates the activity of primary somatosensory cortex in humans," Nature neuroscience, vol. 17, pp. 322-329, jan 2014.

[4] K. Hynynen, N. McDannold, N. A. Sheikov, F. A. Jolesz, and N. Vykhodtseva, "Local and reversible bloodbrain barrier disruption by noninvasive focused ultrasound at frequencies suitable for trans-skull sonications," NeuroImage, vol. 24, no. 1, pp. 12-20, 2005.

[5] J. F. Aubry, M. Tanter, M. Pernot, J. L. Thomas, and M. Fink, "Experimental demonstration of noninvasive transskull adaptive focusing based on prior computed tomography scans," The Journal of the Acoustical Society of America, vol. 113, no. 1, pp. 84-93, 2003.

[6] F. Marquet, Y.-S. Tung, and E. E. Konofagou, "Feasibility Study of a Clinical Blood-Brain Barrier Opening Ultrasound System.," Nano LIFE, vol. 1, no. 3n04, p. 309, 2010.

[7] P. J. White, G. T. Clement, and K. Hynynen, "Longitudinal and shear mode ultrasound propagation in human skull bone.," Ultrasound Med. Biol., vol. 32, pp. 1085-96, jul 2006.

[8] G. Bouchoux, K. B. Bader, J. J. Korfhagen, J. L. Raymond, R. Shivashankar, T. a. Abruzzo, and C. K. Holland, "Experimental validation of a finite-difference model for the prediction of transcranial ultrasound fields based on CT images.," Physics in medicine and biology, vol. 57, pp. 8005-22, 2012.

[9] W. Lee, H. Kim, Y. Jung, I.-U. Song, Y. A. Chung, and S.-S. Yoo, "Image-Guided Transcranial Focused Ultrasound Stimulates Human Primary Somatosensory Cortex," Scientific Reports, vol. 5, pp. 8743-8753, mar 2015.

[10] J. Mueller, W. Legon, A. Opitz, T. F. Sato, and W. J. Tyler, "Transcranial Focused Ultrasound Modulates Intrinsic and Evoked EEG Dynamics," Brain stimulation, vol. 7, pp. 900-908, sep 2014.

[11] G. Pinton, J.-F. Aubry, E. Bossy, M. Muller, M. Pernot, and M. Tanter, "Attenuation, scattering, and absorption of ultrasound in the skull bone," Medical Physics, vol. 39, no. 1, p. 299, 2012.

[12] T. Deffieux and E. E. Konofagou, "Numerical Study of a Simple Transcranial Focused Ultrasound System Applied to Blood-Brain Barrier Opening," IEEE Transactions on Ultrasonics, Ferroelectrics and Frequency Control, vol. 57, no. $12,2010$.

[13] F. Marquet, M. Pernot, J.-F. Aubry, G. Montaldo, L. Marsac, M. Tanter, and M. Fink, "Non-invasive transcranial ultrasound therapy based on a 3D CT scan: protocol validation and in vitro results," Physics in medicine and biology, vol. 54, pp. 2597-2613, may 2009.

[14] R. M. Jones, M. a. O'Reilly, and K. Hynynen, "Experimental demonstration of passive acoustic imaging in the human skull cavity using CT-based aberration corrections," Medical Physics, vol. 42, no. 7, pp. 4385-4400, 2015.

[15] G. W. Miller, M. Eames, J. Snell, and J.-F. Aubry, "Ultrashort echo-time MRI versus CT for skull aberration correction in MR-guided transcranial focused ultrasound: In vitro comparison on human calvaria," Medical Physics, vol. 42, no. 5, pp. 2223-2233, 2015.

[16] M. Wintermark, N. J. Tustison, W. J. Elias, J. T. Patrie, W. Xin, N. Demartini, M. Eames, S. Sumer, B. Lau, A. Cupino, J. Snell, A. Hananel, N. Kassell, and J.-F. Aubry, "T1-weighted MRI as a substitute to CT for refocusing planning in MR-guided focused ultrasound," Physics in Medicine and Biology, vol. 59, no. 13, pp. 3599-3614, 2014.

[17] Q. Grimal, D. Rohrbach, J. Grondin, R. Barkmann, C. C. Gl??er, K. Raum, and P. Laugier, "Modeling of femoral neck cortical bone for the numerical simulation of ultrasound propagation," Ultrasound in Medicine and Biology, vol. 40, no. 5, pp. 1015-1026, 2014.

[18] J. K. Mueller, L. Ai, P. Bansal, and W. Legon, "Computational exploration of wave propagation and heating from transcranial focused ultrasound for neuromodulation," Journal of Neural Engineering, vol. 13, no. 5, p. $056002,2016$.

[19] C. W. Connor, G. T. Clement, and K. Hynynen, "A unified model for the speed of sound in cranial bone based on genetic algorithm optimization.," Physics in medicine and biology, vol. 47, pp. 3925-44, nov 2002.

[20] T. D. Mast, "Empirical relationships between acoustic parameters in human soft tissues," Acoustics Research Letters Online, vol. 7853, no. November 2000, pp. 37-42, 2015.

[21] S. Pichardo, V. W. Sin, and K. Hynynen, "Multi-frequency characterization of the speed of sound and attenuation coefficient for longitudinal transmission of freshly excised human skulls," Physics in Medicine and Biology, vol. 56, no. 1, pp. 219-250, 2011.

[22] B. E. Treeby and B. T. Cox, "k-Wave: MATLAB toolbox for the simulation and reconstruction of photoacoustic wave-fields," Journal of Biomedical Optics, vol. 15, no. 2, pp. 021314:1-12, 2010.

[23] A. Moreira-Gonzalez, F. E. Papay, and J. E. Zins, "Calvarial Thickness and Its Relation to Cranial Bone Harvest," 
Plastic and Reconstructive Surgery, vol. 117, no. 6, pp. 1964-1971, 2006.

[24] L. Ai, J. K. Mueller, A. Grant, Y. Eryaman, and W. Legon, "Transcranial focused ultrasound for BOLD fMRI signal modulation in humans," 2016.

[25] B. E. Treeby and B. T. Cox, "Modeling power law absorption and dispersion in viscoelastic solids using a split-field and the fractional Laplacian," The Journal of the Acoustical Society of America, vol. 127, no. 5, pp. 2741-48, 2014

[26] J. Jaros, a. P. Rendell, and B. E. Treeby, "Full-wave nonlinear ultrasound simulation on distributed clusters with applications in high-intensity focused ultrasound," International Journal of High Performance Computing Applications, vol. 30, no. 2, pp. 137-155, 2016

[27] B. E. Treeby, J. Jaros, D. Rohrbach, and B. T. Cox, "Modelling elastic wave propagation using the k-Wave MATLAB Toolbox," no. 5, pp. 146-149, 2014.

[28] G. Pinton, J.-F. Aubry, M. Fink, and M. Tanter, "Effects of nonlinear ultrasound propagation on high intensity brain therapy.," Medical physics, vol. 38, no. 3, pp. 1207-1216, 2011.

[29] J. L. Robertson, B. T. Cox, and B. E. Treeby, "Quantifying Numerical Errors in the Simulation of Transcranial Ultrasound using Pseudo s pectral Methods," IEEE Ultrasonics Symposium, pp. 2000-2003, 2014.

[30] J. L. B. Robertson, B. T. Cox, J. Jaros, and B. E. Treeby, "Simulation of transcranial ultrasound propagation for ultrasonic neuromodulation and stimulation (Under Review)," Journal of the Acoustical Society of America, 2016.

[31] J. C. Bamber, "Attenuation and Absorption," in Physical Principles of Medical Ultrasonics, ch. 4, pp. 93-166, Chichester, UK: John Wiley \& Sons, Ltd, 2 ed., oct 2005.

[32] P. A. Hasgall, F. Di Gennaro, C. Baumgartner, E. Neufeld, M. C. Gosselin, D. Payne, A. Klingenböck, and N. Kuster, "IT'IS Database for thermal and electromagnetic parameters of biological tissues," 2015.

[33] B. Zurl, R. Tiefling, P. Winkler, P. Kindl, and K. S. Kapp, "Hounsfield units variations: Impact on CT-density based conversion tables and their effects on dose distribution," Strahlentherapie und Onkologie, vol. 190, no. 1, pp. 88-93, 2014.

[34] A. J. Clarke, J. A. Evans, J. G. Truscott, R. Milner, and M. A. Smith, "A phantom for quantitative ultrasound of trabecular bone," Physics in Medicine and Biology, vol. 39, pp. 1677-1687, oct 1994.

[35] D. Nikitichev, E. Alles, S. Noimark, W. Xia, and A. Desjardins, "Acoustic properties of 3D printed materials and their application.," (Under Preparation), 2016.

[36] B. Zeqiri, W. Scholl, and S. P. Robinson, "Measurement and testing of the acoustic properties of materials: a review," Metrologia, vol. 47, no. 2, pp. S156-S171, 2010.

[37] B. Zeqiri, "Validation of a diffraction correction model for through-transmission substitution measurements of ultrasonic absorption and phase velocity," Journal of the Acoustical Society of America, vol. 99, no. 2, pp. 996$1001,1996$. 
(a)

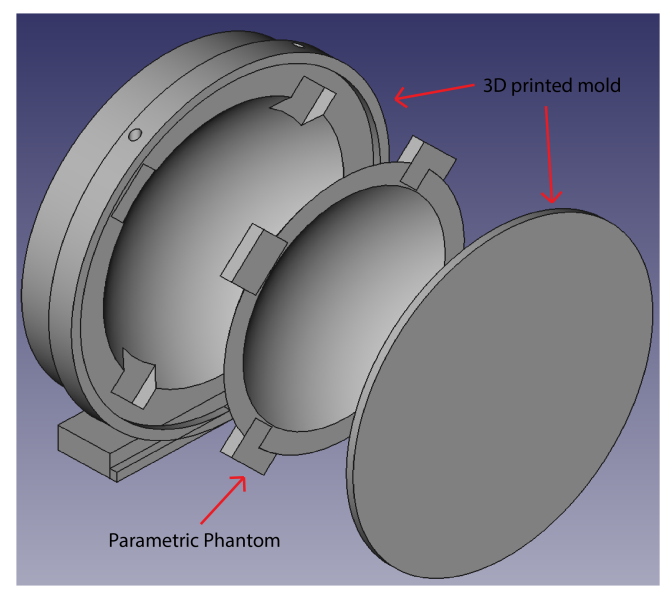

(b)

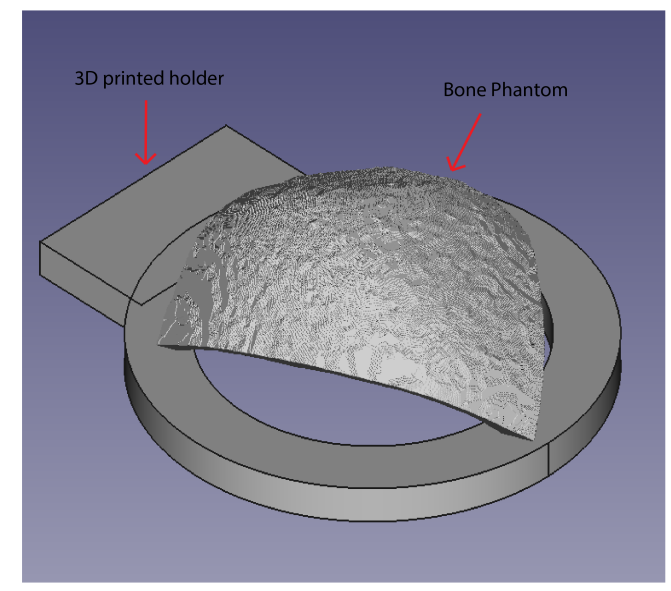

Figure A1: Computer aided design of phantoms. phantom and holder for registration

(a) Parametric phantom and mold. (b) Mesh based

\section{Appendix A. Phantom \& Part Design}

Two skull phantoms were created for the experimental validation described in Sec. 2.6 using rapid prototyping and casting techniques. The first "parametric" phantom was designed to match the idealised skull geometry used in the numerical sensitivity analysis (see Sec. 2.1). This was created by 3D printing a casting mold in a PolyLactic Acid (PLA) using an an Ultimaker 2.0 printer. The mold was then filled with Araldite 1302 using a filling hole, while air was allowed to escape via a vent. The Araldite was prepared by mixing the resin and hardener in the required proportions as specified by the manufacturer, before being degassed in a vacuum chamber, and then injected into the mold. The phantom \& mold are shown in Fig. A1(a). The second "mesh-based" phantom was based on a 3D mesh of the posterior portion of the human skull. This was derived from a T1-weighted MR image obtained from the Imperial College brain development dataset. Brain and skull volumes were extracted using the FSL MRI processing toolbox and converted into a surface mesh using the iso2mesh toolbox. The mesh-based phantom was created in two materials. First, the phantom was 3D printed in VeroBlack using an Objet30 polyjet printer (Stratasys, Eden Prairie, MN). This was then used as a positive to create a vulcanised rubber mold for Araldite resin casting. The phantom is shown in Fig. A1(b). For both phantom geometries, custom holders that allowed the phantom and transducer to be precisely positioned in a pre-defined configuration were created using laser cut PMMA Perspex and 3D printed PLA. This allowed the same alignment to be replicated within the $\mathrm{k}$-Wave simulations. The phantoms and 3D printing files were created using the open source FreeCAD software (http://www.freecadweb.org/).

\section{Appendix B. Material Characterisation}

Two materials were used to create the skull bone phantoms described in Secs. 2.6 and Appendix A, VeroBlack and Araldite 1302 resin. The acoustic properties of VeroBlack were recently reported in [35] thus these measurements were not repeated as part of the current study. However, independent measurements of the sound speed and attenuation of Araldite 1302 resin were conducted. For material characterisation, the Araldite was poured into cuboid rubber molds and left to set. Two samples were constructed, which were then milled to thicknesses of $9.40 \mathrm{~mm}$ and $14.05 \mathrm{~mm}$, measured using a micrometer (Mitutoyo, Kawasaki, Japan). The milling process ensured that the faces of the samples were flat and parallel. Note, the samples 
and resin phantoms were all cast in separate batches.

To characterise the acoustic attenuation and compressional sound speed, through transmission measurements were conducted in a scanning tank. Acoustic waves were generated with a plane piston transducer with an active diameter of $40 \mathrm{~mm}$ (Olympus, Tokyo, Japan), excited with a single cycle toneburst at its central frequency of $1 \mathrm{MHz}$. Time varying pressure signals were recorded with a $0.5 \mathrm{~mm}$ needle hydrophone (Precision Acoustics, Dorchester, United Kingdom). Measurements were repeated at $0.5 \mathrm{~mm}$ intervals along the transducer beam axis between $420 \mathrm{~mm}$ to $580 \mathrm{~mm}$ from the transducer face (to allow field shift compensation, discussed below). For each measurement, the acoustic time-of-flight was calculated from the central maxima and the two adjacent minima of the pulse. Resin samples were then placed between the transducer and the hydrophone. Laser cut PMMA Perspex holders were used to ensure that the samples were orthogonal to the beam axis. The measurements were repeated at $10 \mathrm{~mm}$ intervals along the beam axis between $450 \mathrm{~mm}$ to $550 \mathrm{~mm}$ from the transducer face. Measurements were also repeated with transmission through different positions on the sample, and with the samples at three distances from the hydrophone. Compressional sound speed and attenuation coefficient of the samples were calculated using the through transmission-substitution method outlined in Zeqiri et al. [36, 37]. Frequency dependent transmission loss was calculated by spectrally decomposing the signals and comparing them with free field measurements, selected following field shift compensation based on the measured sound speed.

The measured sound speed and attenuation values are shown in Figure B1. Sound speed values were slightly different for the two samples (which were cast separately) due to minor variations in the Araldite mixture. The mean sound speed of the two samples is $3008 \mathrm{~m} \mathrm{~s}^{-1}$. The corresponding attenuation values are shown in Figure B1(b). As attenuation is calculated through a comparison of the transmission between the two samples, any inter-sample variation in the Araldite mixture could also lead to a systematic error in the calculated attenuation coefficient. The magnitude of this uncertainty is difficult to estimate. The other major source of uncertainty is the measurement of sample thickness. Based on the variation in measurements made at different points on the samples, this is estimated to be no greater than $200 \mu \mathrm{m}$. This uncertainty could lead to a change in sound speed of up to $\pm 56 \mathrm{~m} \mathrm{~s}^{-1}$, and in attenuation of $\pm 0.15 \mathrm{~dB} \mathrm{~cm}^{-1}$. The sound speed of the deionised water is known accurately based on the temperature, while the absorption of ultrasound at frequencies below $5 \mathrm{MHz}$ is negligible [36]. The impact of non-linearity was quantified by examining the spectra of free field measurements taken at the maximum distance from the transducer, and was found to be negligible. Based on the variation in material properties both within and between samples and the uncertainty of the thickness measurements, the estimated uncertainty in the sound speed is $\pm 220 \mathrm{~m} \mathrm{~s}^{-1}$ (or $7.3 \%$ ) and in the attenuation of $\pm 0.6 \mathrm{~dB} \mathrm{~cm}^{-1}$ (or $33.1 \%$ ).

The shear speeds of both Araldite 1302 and VeroBlack were also measured. The same Araldite samples were used, and a VeroBlack sample with a thickness of $6 \mathrm{~mm}$ was $3 \mathrm{D}$ printed using an Objet30 polyjet printer. Shear speed measurements were carried out with a $2.25 \mathrm{MHz}$ shear wave transducer (SWT) and a pulser-receiver (Olympus, Tokyo, Japan). The transducer was excited with a single $2.25 \mathrm{MHz}$ cycle. The time-of-flight of the first and second reflections of the pulse were used with the pulse input time to calculate the shear speed. Results for Araldite resin and VeroBlack are shown in Table 2. Both Araldite and VeroBlack have shear speeds equal to or lower than the speeds discussed in Section 3.1. 
(a)

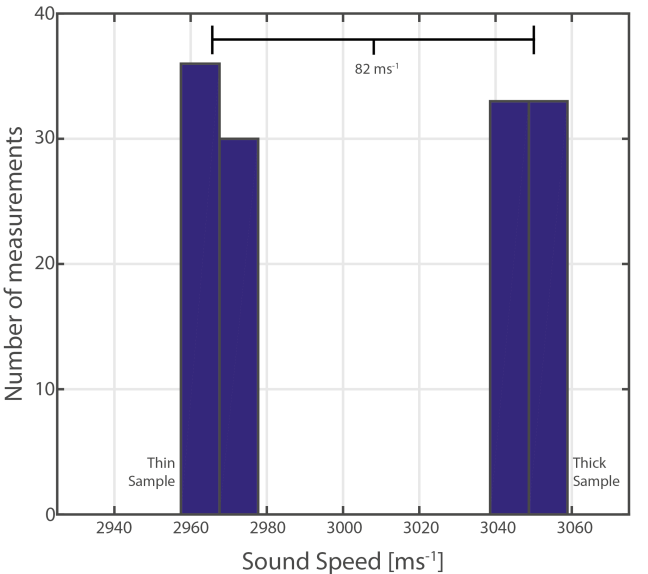

(b)

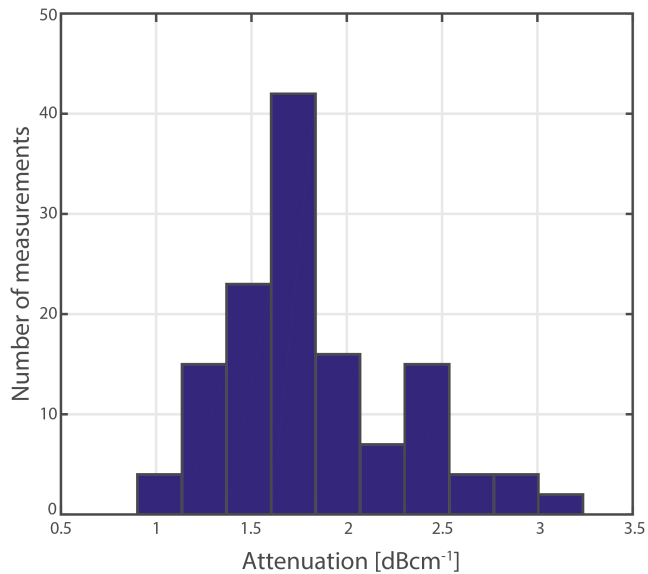

Figure B1: Measurements of the sound speed and attenuation of Araldite 1302 resin. (a) Sound speed, showing the difference between samples. (b) Attenuation values. 